CARNETS DE Carnets de géographes

GÉOGRAPHES.

$7 \mid 2014$

Les espaces de l'entre-deux

\title{
Les minorités tamoules à Colombo, Kuala Lumpur et Singapour
}

Minorités, intégrations socio-spatiales et transnationalités

Delon Madavan

\section{OpenEdition}

Journals

Édition électronique

URL : http://journals.openedition.org/cdg/471

DOI : $10.4000 /$ cdg. 471

ISSN : 2107-7266

Éditeur

UMR 245 - CESSMA

Référence électronique

Delon Madavan, "Les minorités tamoules à Colombo, Kuala Lumpur et Singapour », Carnets de géographes [En ligne], 7| 2014, mis en ligne le 01 décembre 2014, consulté le 24 septembre 2020. URL : http://journals.openedition.org/cdg/471 ; DOI : https://doi.org/10.4000/cdg.471

\section{(c) $(9) \odot$}

La revue Carnets de géographes est mise à disposition selon les termes de la Licence Creative Commons Attribution - Pas d'Utilisation Commerciale - Pas de Modification 4.0 International. 


\section{LES MINORITES TAMOULES A COLOMBO, KUALA LUMPUR ET SINGAPOUR : Minorites, integrations socio-spatiales et transnationalites}

\section{DELON MADAVAN}

La problématique qui sous-tend l'ensemble de cette thèse peut être formulée par une question transversale: comment une minorité diasporique réussit-elle ou non à maintenir la particularité de son identité ethnique et en même temps à être intégrée à la nation dans le cadre spatial des capitales ? L'interrogation sur l'intégration de la minorité tamoule dans des milieux urbains place l'espace au centre d'une recherche qui s'inscrit dans une problématique géographique, bien qu'elle emprunte des méthodes et concepts à d'autres disciplines de sciences sociales. En effet, des notions sociologiques et anthropologiques comme l'identification, la visibilité ethnique ou l'intégration seront considérées en privilégiant l'approche spatiale. Nous pouvons justifier ce choix par le fait que la médiation des flux de population, de la société et de l'espace forme des lieux, des territoires, des paysages et des espaces de vie qui sont autant de formes spatiales de l'identité. Dès lors, l'identité spatiale est un moyen de mesurer et d'analyser la vie, les échanges et les représentations des hommes vivant dans cet espace. Ainsi, avec cette perspective dialectique (flux/société/espace), la démarche géographique vise à démontrer ce que l'espace apprend du maintien ou de l'évolution de l'identité et de l'intégration minoritaire dans la société. En outre, le groupe étudié dans ces trois villes a un statut particulier du fait qu'il est à la fois une minorité ethnique au sein de son pays d'installation, une diaspora pour son foyer d'origine et un des éléments constituant la communauté transnationale tamoule dispersée à travers le monde (Figure 1). C'est pourquoi les échanges transnationaux qui peuvent exister entre Tamouls et les dynamiques migratoires internationales des Tamouls ont également été considérés dans cette étude. 
Figure 1. La communauté transnationale tamoule dispersée à travers le monde

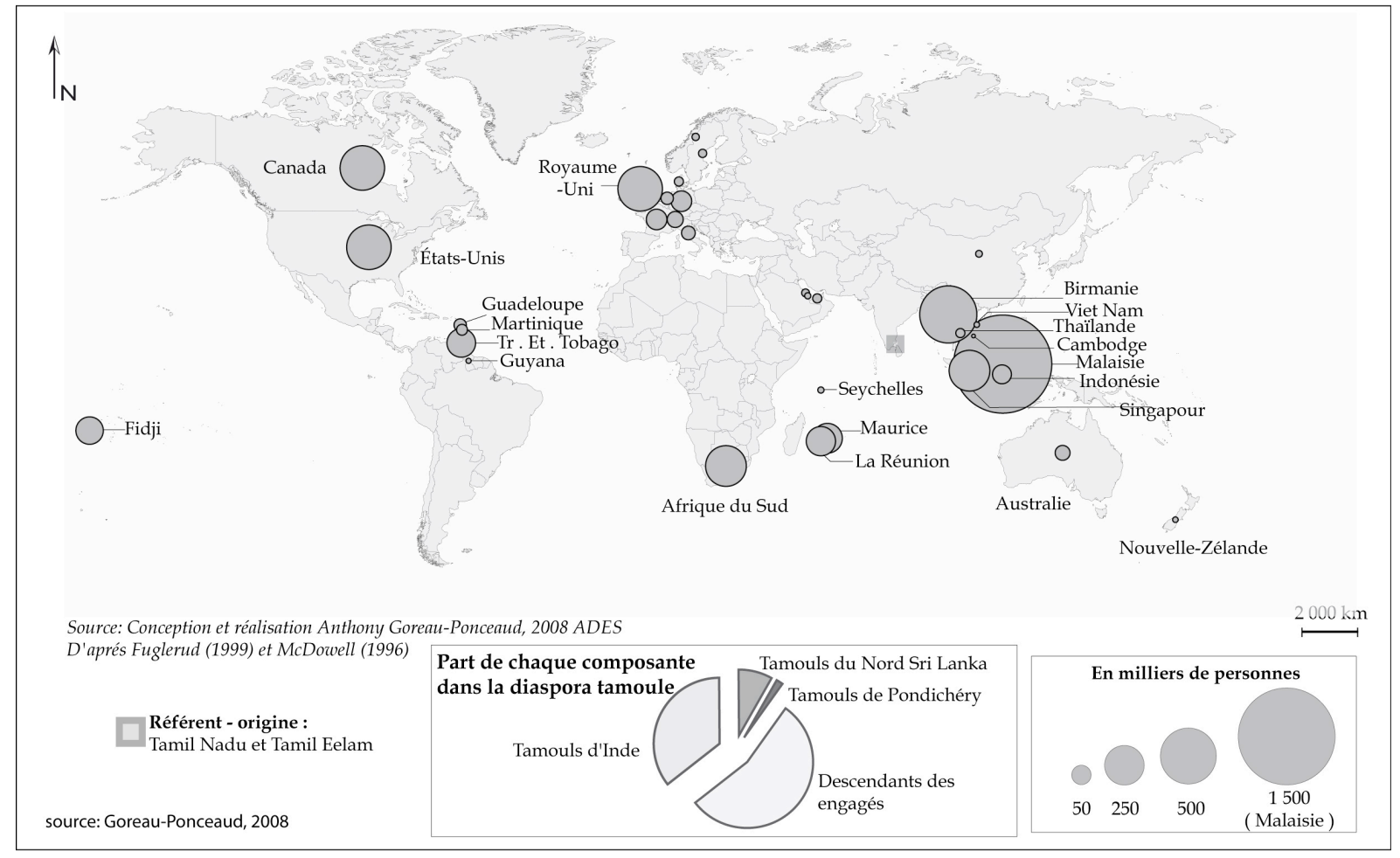

La comparaison de la situation de la minorité tamoule à Colombo, Kuala Lumpur et Singapour permet d'étudier son intégration dans trois États multiculturels post-coloniaux aux contextes politiques nationaux très différents. Ainsi, la situation des Tamouls à Sri Lanka est celle d'une minorité discriminée et dont une faction a été en guerre ouverte contre l'État, alors qu'à l'inverse, à Singapour, la promotion par l'État d'un multiculturalisme reconnaissant quatre identités culturelles (Les Chinois, les Malais, les Indiens et les « Autres ») a empêché la résurgence de conflits inter-ethniques grâce à leur promotion égale. Ce pays semble réussir à concilier un haut niveau d'ethnicité, c'est-à-dire la reconnaissance de la singularité des communautés à travers certains marqueurs culturels comme la langue, la religion ou la région d'origine, avec une identité nationale forte. Les Tamouls de Malaisie évoluent pour leur part dans un contexte dans lequel les populations bumiputeras sont privilégiées. En effet, l'État y conduit une politique officielle de discrimination positive favorisant la population malaise au détriment des minorités chinoises et indiennes. Ces trois exemples différents et complémentaires présentent l'avantage d'apparaître comme emblématiques de la situation des minorités en général.

L'analyse des politiques menées par la puissance coloniale, puis par les trois États indépendants à l'égard des minorités, permet dans la première partie de mieux comprendre leurs impacts sur le sentiment d'identification et d'intégration des Tamouls à la nation, ainsi que sur l'évolution de leur distribution dans les villes étudiées. Une enquête de terrain socioanthropologique (questionnaires, enquêtes au fil de la rue et travail d'observation des espaces publics et domestiques), dans dix quartiers habités ou fréquentés par des Tamouls (Figure 2), a permis d'examiner à l'échelle locale les fondements territoriaux de l'identité tamoule, d'analyser la place de la culture tamoule et la façon dont les membres de cette communauté vivent et s'identifient. Les pratiques citadines des Tamouls, notamment celles qui ont trait à la 
maison et à la fréquentation des lieux de sociabilité communautaires (les édifices religieux, les commerces et les associations), peuvent également être à l'origine d'une territorialisation de l'identité tamoule dans ces villes. En outre, les politiques urbaines (éradication des bidonvilles, gentrification et promotion de Little India) adoptées par les deux États Sud-Est asiatiques ont des conséquences sur l'empreinte urbaine tamoule, sur le sentiment d'intégration des membres de cette minorité et leurs pratiques urbaines. Enfin, dans la troisième partie, l'étude des liens transnationaux (culturels, politiques, économiques) entre Tamouls et les dynamiques migratoires internationales contemporaines des Tamouls dans ces trois pays a permis de montrer que ces derniers ont également des conséquences sur l'identification et l'intégration de ceux de Colombo, Kuala Lumpur et Singapour. 
Figure 2 . Les terrains étudiés à Colombo, Kuala Lumpur et Singapour

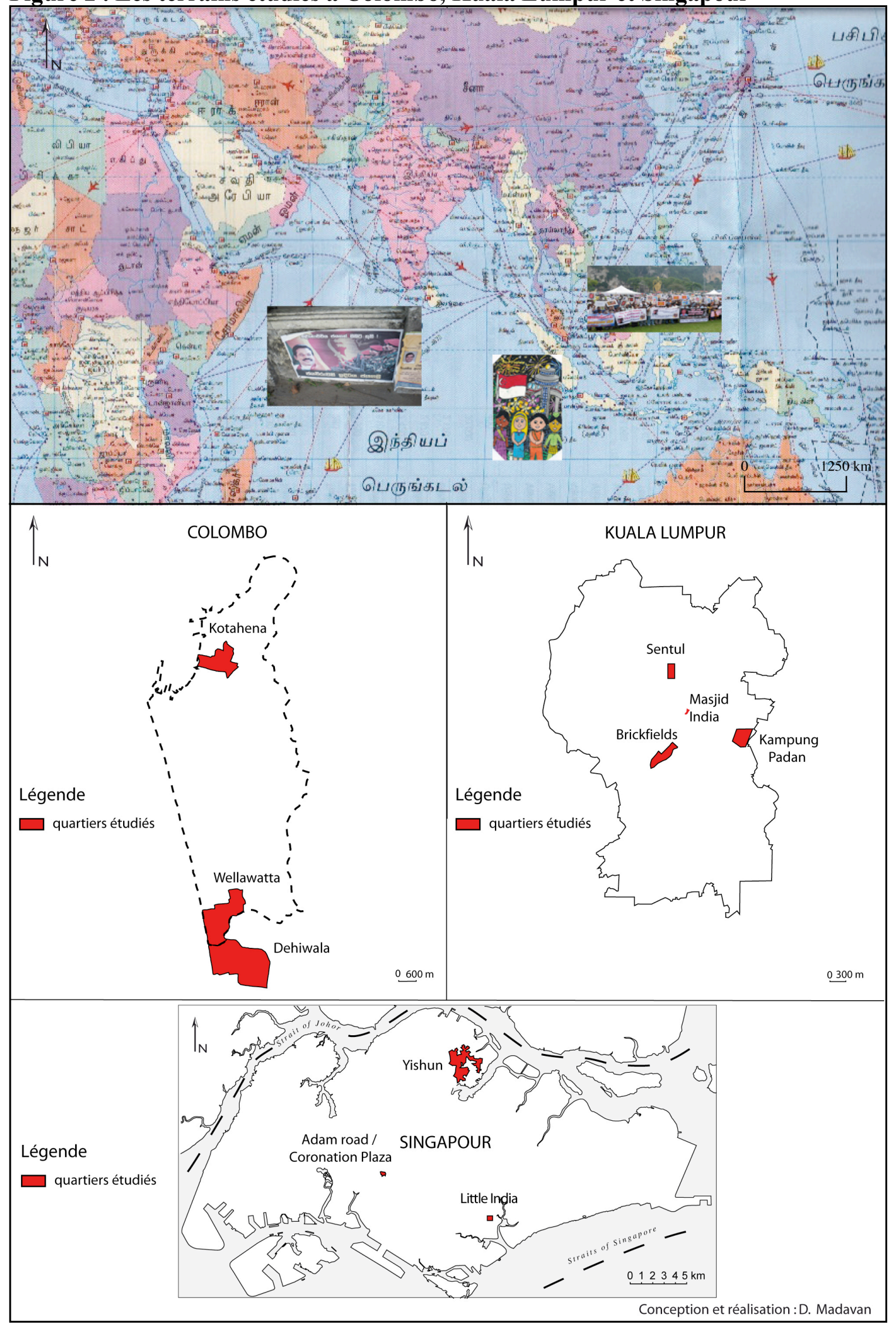


L'étude des Tamouls dans ces trois villes démontre donc l'intérêt d'adopter une perspective comparative et multiscalaire pour saisir dans toute leur complexité les différents facteurs agissant sur l'identification et l'intégration socio-spatiale d'un groupe minoritaire transnational. Dans le cas de minorités ethniques d'origine diasporique telle que celle des Tamouls, la politique d'intégration de l'État d'installation, l'inscription spatiale de leur identité, les pratiques au sein de l'environnement urbain, les liens officiels ou informels qui sont préservés avec le pays d'origine, et les solidarités ethniques infra et transnationales, participent à des degrés différents à la construction d'une identité communautaire qui peut, selon le contexte historique, économique et l'environnement socio politique, cohabiter ou concurrencer l'identité nationale.

Fiche informative

\section{Discipline}

Géographie

\section{Directeur}

Olivier SEVIN, Professeur, Université Paris-Sorbonne

\section{Université}

Univeristé Paris-Sorbonne

Membres du jury de thèse, soutenue le 26 septembre 2013

- Christian HUETZ DE LEMPS, Professeur émérite, Université Paris-Sorbonne

- Frédéric LANDY, Professeur, Université de Paris Ouest Nanterre La Défense

- Éric MEYER, Professeur émérite, INALCO

- Chee WONG TAI, Professeur, Nanyang Technological University (Singapour)

- Olivier SEVIN, Professeur, Université Paris-Sorbonne

\section{Situation professionnelle actuelle}

À la recherche d'un poste et qualifié en section $23 \mathrm{du} \mathrm{CNU}$

\section{Courriel de l'auteur}

delonmadavan@gmail.com 